\title{
UNA VISIÓN INTEGRAL DEL EMPRENDEDOR, DESDE UN CONTEXTO REGIONAL CULTURAL, ORIGINADO POR LA FENOMENOLOGİA
}

\section{A COMPREHENSIVE VISION OF THE ENTREPRENEUR, FROM A REGIONAL CULTURAL CONTEXT, ORIGINATED BY PHENOMENOLOGY}

Fernando Adolfo Fierro Celis*

\begin{abstract}
Resumen
Cuando se realiza el emprendimiento en el país, se utilizan las mismas acciones y metodologías en las diferentes instituciones, sin tener en cuenta la caracterización de las regiones y de las personas. Esto se presenta porque se parte del hecho que los individuos que forman una comunidad poseen la misma forma de pensar y actuar frente a un tema en específico, sin embargo se nota claramente el desarrollo del emprendimiento en unas regiones más que otras.

Este fenómeno se puede explicar desde como la fenomenología forma la cultura de los individuos, y esta moldea la personalidad que converge en el desarrollo de competencias, que cambian el contexto del emprendedor, al revisar este fenómeno es importante la formación como eje central. Todos nacemos emprendedores, pero la educación facilita el proceso de cristalizar la idea, sin embargo esta educación debe estar orientada teniendo en cuenta el tipo cultural de la región.
\end{abstract}

Palabras clave: fenomenología, cultura, entorno, personalidad, competencia, educación.

\section{Abstract}

When performing entrepreneurship in the country, the same actions and methodologies are used in different institutions, regardless of the characterization of the regions and people. This occurs because it is assumed that individuals within a community have the same way of thinking and responding to a specific topic, however, the development of entrepreneurship is clearly seen in some regions more than others.

This phenomenon can be explained by understanding how phenomenology is part of the culture of individuals, and how it shapes the personality that converges in the development of skills that change the context of the entrepreneur; in reviewing this phenomenon it is important to consider training as a central axis. We are all born entrepreneurs, but education facilitates the process of crystallizing the idea, however this education should be oriented according to each region.

Key words: phenomenology, culture, environment, personality, competence, education.

Artículo recibido: 20/06/2013 Aprobado: 15/07/2013

* Estudiante de Doctorado en Gestión. Docente Facultad de Economía y Administración. Email: ffierro@usco.edu.co 


\section{Introducción}

Hay países que se han desarrollado más que otros siendo inclusive del mismo continente, es más, estados dentro de un mismo estado, que se han diferenciado. La inquietud que surge es a que se debe este desarrollo, varios estudios y autores han esbozado este suceso a diferentes factores tales como ubicación, cultura, personas entre otros, sin embargo existe un factor común en donde converge el cual es el ser humano como emprendedor bajo un contexto cultural.

Aunque son importantes no se ha podido caracterizar dentro de un marco que nos ayude a delimitar los procesos académicos o no convencionales que sea más didáctico el desarrollo de la creación de empresas, esto ha llevado a muchos investigadores en esta área a centrarse en el estudio de variables más dinámicas y modelos que tuvieran en cuenta no solo aspectos personales, sino también sociales, así como la interacción entre ambos para explicar y predecir la conducta emprendedora. (Alexei y Kolvereid, 1999; Audet, 2002; Crant, 1996; Douglas y Shepherd, 2002; Krueger, Reilly y Carsrud, 2000).

Como es bien sabido el emprendedor en primera instancia se debe enfrentar a diferentes escenarios en su proceder de construcción empresarial, como se encuentra en palabras de (Fayole y Bruyat, 2002) señala que los economistas han tendido a aproximarse al tema desde una visión funcional del fenómeno centrada en el rol del emprendedor («el qué»); las ciencias humanas han puesto el énfasis en los aspectos personales («quién» y «por qué»), mientras que las ciencias de la gestión y la organización se han centrado en el proceso («el cómo»). Bajo este esquema el enfoque que se pretende abordar es el de la cultura desde la fenomenología y el de las ciencias humanas, el cual coloca importancia en el quien y el por qué.

\section{La realidad y la fenomenología en el emprendedor}

Para analizar el proceso de la cultura en una sociedad y el ser humano dentro de ella, es importante conocer la fenomenología donde esboza que «Desde un enfoque filosófico, expresado en el pensamiento de Husserl, explicitar la correlación entre el hombre, su realidad y el mundo de las cosas, es decir, la estructura de la experiencia humana, es lo que se ha llamado fenomenología (herrera, 1998, p. 50).
Una vez más para la fenomenología, la cultura es el conjunto de producciones que tienen lugar en las actividades continuas de los hombres colectivizados, que tiene su existencia espiritual permanente en la unidad de la conciencia de la comunidad y que su tradición sigue conservando (Hoyos y Vargas 1996, p. 100) de esta manera se hace más fácil la comprensión de la interacción de la sociedad y el individuo en la construcción del contexto cultural.

Esto conlleva a determinar que el conjunto de producciones que se emanan entre la correlación entre el hombre y la realidad, crean nuevos parámetros en las diferentes sociedades, lo que indica que cada región de acuerdo a su forma de cómo fue fundada, por quienes fueron fundadas o como se desarrollaron. Crean diferentes culturas (realidades) que les enseña la mejor forma de cómo abordar diferentes temas, porque sencillamente su colectivo de individuos está familiarizado con situaciones inherentes a la gran mayoría de personas de cierta sociedad.

Al analizar el fenómeno del emprendimiento dentro de una realidad regional, se tiene como factor transversal al ser emprendedor, el cual crea cultura y «la cultura constituye una variable importante» Orrego (2009, p. 24) tanto para el desarrollo de la sociedad como del individuo, sin embargo la respuesta de una sola persona a la problemática de desarrollo no es suficiente se deben, «construir comunidades de voluntades de personas determinadas que están de acuerdo, incluso inmediatamente, como sujetos volitivos» Husserl 1987, (p. 183) para que de esta manera la influencia de una persona se vea reflejado en las demás y sea más fácil el poder permear al sujeto en todas sus expresiones.

Es importante recordar que así como el ser humano es base para la creación de la cultura o una nueva sociedad, cada sujeto interpreta y da su propio significado a su existencia. Bajo este esquema Husserl afirma: «yo, significa para cada uno de nosotros algo diferente: para cada uno Yo, significa la persona completamente determinada con un nombre propio concreto, que vive sus percepciones, recuerdos, expectativas, fantasías, sentimientos, deseos, voliciones, que tiene sus estados, ejecuta sus actos, y además, tiene sus disposiciones, predisposiciones innatas, capacidades y habilidades adquiridas» (Husserl, 1994, p. 48) colocando una vez más el ser humano en toda su comprensión.

Al encontrar un punto en común de cada individuo en una sociedad se puede unificar formas de pensar que 
reflejan las creencias de una sociedad, parafraseando a Herrera, (1998) «el ser humano es un ser cultural, que experimenta el mundo social en termino de motivaciones; de acuerdo con esto, desempeña ciertos comportamientos, se ajusta a valores, y especialmente se dispone creativamente para emprender como un acto de sentido; a su vez, se siente determinado por las valoraciones positivas o negativas e influenciado por sus coterráneos.

Bajo los parámetros anteriores se puede determinar que cada sociedad crea su propio concepto de individuo y sus propias características (en este caso del emprendedor), que se fortalece en el esquema mental Senge, (2005) de cada persona. En este sentido Husserl afirma que «los seres humanos deben comprometerse a configurar una cultura auténticamente humana»

Sin embargo esta cultura o desarrollo de la misma desembocan en la definición de sujeto como emprendedor, se influencia por la comprensión de la realidad que cada individuo tenga, bajo este concepto Herrera (1998, p. 18) comenta que «es a través de nuestra experiencia que la realidad adquiere un sentido» y esta realidad se construye a través de los conocimiento que se adquieren durante el tiempo. Es decir cada departamento del país posee experiencias diferentes de crecimiento que de manera inconsciente, le dice cuál es la mejor manera de desarrollar su región debido a sus características propias e inherentes.

Para complementar la realidad de cada individuo Schutz $(2003$, p. 20) comenta que «cada sujeto comprende su realidad y su sentido común, toda vez que cada individuo integra un mundo particular, en el cual no solo hay objetos sino otros semejantes» en donde se puede validar que la cultura y la sociedad influye en el concepto y la forma de ver, lo que queremos ver.

Al esbozar el termino de realidad según Schutz no podemos dejar de lado la importancia que toma el sujeto dentro de esta definición, continuando con el autor afirma: «no puedo comprender una cosa social sin reducirla a la actividad humana que la ha creado, y, más allá de ello, sin referir esta actividad humana a los motivos que la originan» $(2003$, p. 23) es decir el sujeto es parte vital de toda sociedad y el mismo crea su propia definición de sujeto como emprendedor, de acuerdo a la experiencia de él, y de la sumatoria de las demás experiencias de toda una comunidad, por esta razón cada región tiene diferentes experiencias, teniendo la misma necesidad (el desarrollo del emprendedor), lo que obliga el abordaje de la educación del emprendimiento de diferente manera para cada uno de ellos.

\section{El entorno y su contexto regional}

Una vez, creada la realidad inmersa en una cultura, que identifica a todos los individuos de un colectivo, se origina el contexto regional. De hecho, los territorios se desarrollan a partir de contextos únicos definidos por una configuración industrial, histórica y local, así como por el patrón de inversión en recursos que se efectúa con el tiempo (Auerswald \& Branscomb, 2003).

De manera particular, permite explicar las ventajas que se poseen, las cuales son específicas de un territorio y tienen que ver tanto con la posesión como con la capacidad de adquirir o desarrollar un conjunto de recursos que los competidores 0 áreas rivales no poseen (Wernerfelt, 1984; Barney, 1991). Es decir, así como cada persona mejora su posición, lo debe hacer cada región para el mejoramiento de su entorno, se puede afirmar que el entorno en sí mismo contiene y, al mismo tiempo, apoya la actividad empresarial Neck et al., (2004).

Este desarrollo de contextos diferentes para cada región, de acuerdo a su evolución, crea activos propios a los recursos, que ninguna otra región los tiene, estos activos tangibles e intangibles que condicionan la creación de empresas, aportan las bases que permiten diferenciar, desde una perspectiva dinámica, aquellos territorios que tienen un grado diferencial de éxito al estimular el desarrollo de dichas empresas (West, Bamford \& Marsden, 2008). Y, debido a la diversidad de recursos que poseen los departamentos del país, cada uno de ellos como su gente, es apto para desarrollar diversas de formas de emprendimiento.

Para profundizar un poco más, el entorno, comprende un conjunto de variables que generan oportunidades y amenazas para las actividades de emprendimiento. Hugo Kantis, en su texto Desarrollo emprendedor: América Latina y la experiencia internacional, lo define como el sistema de desarrollo emprendedor, que tiene como componentes las condiciones sociales y económicas, los aspectos culturales y el sistema educativo, la estructura dinámica y productiva, los mercados de factores, las redes - sociales, productivas e institucionales-, los aspectos personales y las regulaciones políticas. Cada uno de los 
factores que se mencionan anteriormente se desarrolla en diferente escala, en las diferentes zonas, lo que indica que en cada una de ellas, el énfasis debe ser diferente y no general para los territorios.

Sin embargo, es importante resaltar que debido a la diversidad cultural de nuestro país, cada región puede mejorar o cambiar su realidad (cultura) para desarrollar una ventaja competitiva de la empresa sobre la base de la combinación y utilización de los recursos de los que esta dispone o puede desarrollar (Foss et al., 2007). Aunque es importante, mencionar que estos recursos son sistémicos y pueden ser duraderos a través del tiempo (West \& Bamford, 2005; West, Bamford \& Mar- sden, 2008). Aumentado o disminuido por los recursos específi$\cos$ que posee un territorio, sus recursos naturales (por ejemplo su clima), como los recursos creados por la comunidad, ya sean de uso generalizado (carreteras) 0 especializado (institutos de investigación específicos) (Porter, 1991).

La combinación adecuada de recursos avanzados y especializados de naturaleza, principalmente, intangible, la correcta movilización de los mismos a través del establecimiento de múltiples interacciones entre ellos y la dificultad de su adquisición, imitación y sustitución conlleva, justifican el fracaso de ciertas iniciativas para desarrollar ecosistemas emprendedores, incluso aunque hayan estudiado la estructura y conjunto de recursos de un área modélica (West \& Bamford, 2005). Cada zona debe educar a sus posibles emprendedores de acuerdo a sus propias limitaciones y no haciéndolo por regla general, de esta manera se disminuye el fracaso en los intentos de emprendimiento.

La afirmación de Neck et al. (2004) respecto a la necesaria evolución temporal de ciertos componentes que interactúan para formar un sistema dinámico que alimente la creación de empresas, Cada región o país deberá, a partir de su combinación actual de recursos y establecimiento de una meta a alcanzar a largo plazo, trazar su camino para adquirir aquellos recursos nuevos o que complementen los existentes y que sean especialmente atractivos para el sistema, lo cual desarrollaría, de una forma gradual, su propio entorno emprendedor sostenido por ventajas en propiedad idiosincrásicas (West, Bamford \& Marsden, 2008).

El fin principal de tener variables eficientes que permitan permear todos los sectores de la economía, es que los individuos de un territorio aumenten las posibilidades que poseen los sujetos de tener oportunidades de desarrollar procesos productivos, que les permitan generar su propio empleo y riqueza, consintiéndoles además mejorar su calidad de vida y proyectarse a futuro como generadores de valor agregado a todos los procesos que hacen parte del funcionamiento económico y social de la comunidad Amartya Sen, (1998).

Continuando con el análisis de Sen (1998), «La meta del desarrollo humano es la libertad, el desarrollo de la comunidad se mide por el grado de libertad que tenga», bajo este esquema, se podría afirmar, que una buena maduración de las variables que influyen el entorno, otorgan a los territorios en que se implantan un elevado grado de competitividad y crecimiento económico, al contribuir con la eficiencia estática y dinámica del sistema económico (Colombo \& Grilli, 2005), en ese sentido, Stevenson (2000), plantea: «el entorno es importante, y es más factible que un individuo pueda comenzar a tener actitudes emprendedoras si actúa en un contexto en el que se facilita el reconocimiento de la oportunidad y su persecución».

Cada región es un contexto social dinámico, que actúa de acuerdo a las variaciones del tiempo y de los recursos que se deriva de la zona, esto conlleva al cambio de mentalidad o formación de la personalidad del individuo dentro de su propia realidad, Según Mucchielli $(1985,145)$, la mentalidad se puede definir como «un sistema de referencia implícito de una sociedad o grupo social, homogéneo desde el punto de vista del espíritu común. Este marco de referencia permite a las personas percibir e interpretar la realidad de cierta manera y por tanto ver las reacciones y conductas de acuerdo con esa percepción del mundo», o de su zona, colocando la necesidad de cambiar personalidades mediante la educación enfocada a las realidades de cada territorio.

\section{La realidad del locus del control interno}

Al tener como base la persona y su comportamiento, y en aras de entender el proceso de como la realidad afecta la personalidad y genera rasgos se tiene como base, el locus del control interno, en palabras de Robbins, (1999) en donde nos orienta que la percepción que una persona tiene sobre la fuente de su destino, se conoce como locus de control interno. Es decir la persona donde nace y desarrolla su forma de pensar ya está sentenciado a actuar 
y tener esquemas mentales senge, (2005) que lo hacen creer que esa predestinado de acuerdo a su región.

Bajo otro punto de vista se encuentra (Ong e Hishamuddin, 2008) en donde plantea que el control interno se define como la creencia que tiene una persona sobre los resultados, a través de la habilidad, el esfuerzo o las destrezas que posee, y no tanto sobre las acciones que otros llevan a cabo. Bajo este esquema se puede inferir que cada individuo es dueño de sus decisiones y de su propio resultado, es decir cada uno de manera consciente supone que construye su propio mundo y este mundo es el mejor para la persona, pues solo conoce este. Sin embargo estas decisiones están influenciadas por las oportunidades que brinda su entorno, de esta manera cada individuo desarrolla habilidades de acuerdo a su zona.

Así lo argumentan también Miller y Toulouse (1986), al decir que, una persona interna cree que las consecuencias de su conducta provienen de sus propios esfuerzos, y una persona externa considera que los eventos de su vida dependen de factores que están fuera de su control, atribuibles a la casualidad 0 al destino. Bajo este esquema los factores externos son las realidades 0 la cultura que rodea al individuo para el desarrollo de su potencial como emprendedor, de esta manera se puede influenciar al individuo a través de la educación, para el fortalecimiento del locus, orientado hacia el emprendimiento con las limitaciones que brinda el entorno.

Una vez el individuo de manera consciente 0 inconsciente (Popper), crea rasgos psicológicos que caracterizan a su región o la región crea rasgos que caracterizan al individuo, de esta manera, uno de los principales factores que se debe tener en cuenta es, que tanto se conoce el individuo (en términos de capacidades y habilidades) para comenzar a desarrollar su papel dentro de la sociedad, en palabras de Dyer (1994), argumenta que partiendo de la aceptación del papel básico que estas personas desarrollan en el entorno social, es evidente que a medida que se conozcan mejor los rasgos personales que definen al emprendedor, se estará en una posición óptima para promover las iniciativas. Este papel, como lo explica Dyer (1994) lo da cada región y por ende al revisar este proceso no se puede evaluar que tan consciente 0 volitivo es la persona para este Proceso, o que tantas oportunidades le brinda el limitado entorno.

Tal como lo indica Dyer, (1994) exponiendo que las características más importantes para que los jóvenes es- tudiantes muestren una inclinación hacia el emprendimiento, se encuentran los rasgos psicológicos, estos rasgos determinan en muchas ocasiones el comportamiento del emprendedor que son traducidas a la actividad emprendedora en actitudes, en palabras de Robinson et al. (1991), las actitudes, realidades y educación determinan realmente el perfil emprendedor.

Cuando se empieza a configurar la teoría de rasgos y, al tener como expositor principal a David Mcclelland (1961) se converge una idea entre los investigadores que es muy similar de que los emprendedores generalmente poseen las características, necesidad de logro, creatividad e imaginación, su propia confianza en las capacidades que poseen así como una gran capacidad de negociación y alta propensión al riesgo (Green et al., 1996).

Así mismo, Korunka, et al. (2003), han estudiado las características de la personalidad de nuevos empresarios. Consideran que la personalidad del emprendedor debe tener ciertos rasgos o características específicas.

Esta influencia se puede ver reflejada, en la investigación que realizado Korunka et al. (2003), a 1,169 empresarios, respecto al control interno, encontraron que los empresarios con éxito tienen un alto grado de control interno, es decir existe relación entre el locus del control interno que se reflejan en las conductas del emprendedor. A su vez Moriano et al. (2004), mencionan que los emprendedores tienden a atribuir los éxitos o fracasos a causas internas de su conducta. Y como la conducta son comportamientos habituales y estos comportamientos que se basan en aptitudes 0 habilidades se pueden aprender (Veciana, 1999), el posible emprendedor tiene la posibilidad de influenciar su realidad, para cambiar su personalidad y alcanzar el éxito en su emprendimiento.

\section{De las competencias del entorno, a las competencias del individuo}

Una vez desarrollada la personalidad, esta se encarga de que el individuo sea apto para el desarrollo de sus competencias, y estas se encargan de que Toda persona que ingresa al mundo laboral (indiferente su posición de empleado, empresario o líder) se enfrenta a la empleabilidad del mercado que está regida por un término que marca la pauta en el proceder de las personas, «La competencia laboral es el elemento operativo que vincula la capacidad 
individual y colectiva para generar valor con los procesos de trabajo, por lo que constituye una nueva alternativa para mejorar el rendimiento de los individuos y de la organización» (Sánchez, Marrero, Martínez, 2005. p. 1), en términos de emprendimiento es como la capacidad de estos individuos proceden con las necesidades del medio o del entorno para satisfacerlas en términos de gestación (emprendimiento) y desarrollo (Liderazgo) de las empresas.

Continuando con el término de las competencias, están enmarcadas en «tres grandes tendencias o corrientes en el estudio de las competencias identificadas por Mertens» (1996). En primera instancia esta la corriente conductista, (Sánchez, Marrero, Martínez, 2005. p. 3). La segunda corriente, la funcionalista, toma como base dos factores en el desarrollo de la escuela; la persona y el puesto 0 las funciones que tiene este puesto, como señala Mertens (1996). Como última corriente se encuentra conformada en Francia, tiene su principal exponente en la autora Claude Levy-Levoyer (1997) quien plantea que «las competencias son una lista de comportamientos que ciertas personas poseen en mayor medida que otras y que las transforman en más eficaces para una situación dada.

Una vez revisada las «tres grandes tendencias 0 corrientes en el estudio de las competencias identificadas por Mertens» (1996). Se encuentra que la corriente que más se aproxima al emprendimiento 0 al proceso de desarrollo del emprendedor, es la corriente conductista, el cual se enfoca al cambio conductual, fenómeno que le permite al emprendedor (Gilder, 1984), lograr sus objetivos, quebrando viejos patrones y creando unos nuevos, estos patrones en primera instancia son creados por la femonología, de esta manera se explica que cada individuo de cada zona, posea unas capacidades 0 competencias diferentes a individuos de otros territorio.

Continuando con la corriente conductista, se encuentran características, capacidades y habilidades del empresario. En palabras de Varela. (2008, p. 142) realiza un cuadro con base en investigaciones y resume las características empresariales en: factores motivacionales, características de comportamiento, características individuales. Todas estas características influyen directamente el proceder del emprendedor y están arraigadas en mayor o menor actuación en los individuos dependiendo del origen de su departamento.

Por otro lado Gibbs (2005) planteo una serie de valores empresariales que le permiten al empresario hacer, orga- nizar, sentir, comunicar, entender, pensar, y aprender cosas vitales para su desarrollo. Mientras tanto Timmons hace una integración diferente y plantea seis grandes categorías las cuales son: compromiso y determinación, obsesión de oportunidad, tolerancia al riesgo, ambigüedad e incertidumbre, creatividad, adaptabilidad, autoconfianza.

Según Varela y Bedoya (2008. P. 149) indica que las competencias empresariales son el conjunto de atributos (motivaciones, actitudes, valores, conocimiento, habilidades) de una persona, que se manifiestan en comportamientos definibles, observables medibles, que están vinculados, en forma casual, con un desempeño superior en la acción empresarial

Como se analiza en los planteamientos de las competencias de los diferentes autores como Varela, Gibbs, Timmons y Bedoya, se puede observar que todas las competencias mencionadas anteriormente, las desarrollan los individuos de acuerdo a lo que ellos creen y el entorno que le brinda las oportunidades o limitaciones, o su propio éxito. En La escuela de Mc Clelland (Del Pino, 1997) pone el acento en las entrevistas, en la persona, para llegar a perfilar la «conducta situacional» de los que triunfan o fracasan, lo que nos indica que estos cambios de conducta situacional (desarrollados por su cultura) afectan el perfil de las personas en el desarrollo de los sucesos para emprender, por ende la educación orientada según la región, es un factor transversal para el desarrollo sostenibles del emprendimiento priorizado.

\section{La educación dentro de cada contexto}

Para la persona como sujeto emprendedor, existe la formación entendida como la estructura o mejoramiento del ser, en palabras de Vargas (2007, p. 26) La formación requiere no solo el reconocimiento del carácter anónimo constitutivo del sujeto sino de la cultura, es decir, se trata de «(...) comprender las estructuras fundantes de la interacción mundano-vital; tal transito considera tanto la formación de las personas singulares como el fundamento de las personalidades de orden superior-familia, comunidad, barrio, ciudad, nación, estado, comunidad de naciones». Es a través de todas las realidades y experiencias que el individuo fortalece sus valores que incrementaran la probabilidad de escoger el camino del emprendimiento.

Además existen otros actores para el mismo escenario, parafraseando a Orrego $(2009$, p. 28) «el emprendimiento 
se explica no solo a partir del autoconocimiento, la autovaloración, la autodeterminación de los sujetos emprendedores, sino también del papel que juegan los entes 0 personalidades de orden superior que interviene en el mundo social, como son el estado, la organización, la familia y las comunidades, entre otros, y la comprensión de la interacción que genera el emprendimiento como posibilidad de realización del sujeto en la vida con los otros» lo que nos indica que el ser emprendedor no se hace de la noche a la mañana es un proceso integral al ser humano que se fortalece y está ligado a su contexto, a la formación del individuo dentro del contexto y a las oportunidades que brinda.

La preocupación por lograr una educación emprendedora tiende a estar cada vez más integrada en la agenda de los gobiernos e incluye acciones que van desde la escuela primaria hasta la universidad. Hugo Kantis, América Latina y la experiencia internacional. $2004(p, 135)$. Dether (2001:5) lo afirma: «... Nacemos emprendedores, pero la educación puede facilitarnos el proceso de materializar nuestras buenas ideas en todos los campos de nuestras actividades intelectuales y profesionales, en tanto «nos hace» mejorar nuestras actitudes y aptitudes para emprender» de acuerdo a su contexto.

Continuando con la importancia de la educación como lo plantea Gibb, «la educación empresarial debe ser integral y no fragmentada, debe analizar en forma integral, pragmática y racional los diversos elementos de la actividad empresarial mirando las sinergias que puedan existir», estas sinergias están dadas por la interacción de las experiencias de los individuos que están marcadas por su propia realidad.

A su vez ronstadt plantea, entre otras, la siguiente idea sobre educación empresarial: «la carrera empresarial es un proceso, con muchas oportunidades de empresa, en el cual existan muchas formas para configurar y ejecutar una nueva idea, la cual por otro lado, está siempre sujeta a mejoras, un proceso donde el tiempo es el factor limitante no solo en términos del inicio y de la ejecución, sino también en términos de planeación e investigación de nuevas oportunidades»

Varela y Bedoya plantea que la formación de un empresario es un proceso en el cual intervienen un sinnúmero de variables sociales, culturales, psicológicas y económicas que contribuyen, con un conjunto de conocimientos específicos, a desarrollar una serie de competencias cuyo propósito es lograr que este empresario es formación tenga altas probabilidades de convertirse en un empresario exitoso, capaz de generar riqueza y desarrollo social a lo largo de su vida.

Diferentes estudios han mostrado que la educación puede estimular el desarrollo de la conducta emprendedora de diferentes formas. Por una parte, la educación para el autoempleo puede incrementar los conocimientos sobre creación y gestión de empresas, y fomentar características personales asociadas con los emprendedores como la motivación de logro, el locus de control interno o la autoeficacia (Bonnett y Furnham, 1991; Gorman et al., 1997; Hansemark, 1998; Krueger y Dickson, 1994; Rasheed, 2003), sin embargo esto significaría el cambio en el esquema académico de todo un país para la enseñanza del aprendizaje.

Como se puede observar con los autores expuestos, ninguno pone en evidencia el contexto para el desarrollo del emprendedor ni como esto influye, el tema de fondo consiste en comprender los efectos de estas variables en la realidad de cada país y de cada sector donde se quiera fomentar actividades de emprendimiento. De esta forma podrán elaborarse planes de negocios bajo un marco coherente y será viable proponer acciones gubernamentales para apoyar a los emprendedores. Duarte. (2007, p. 48).

La educación es fuente principal del desarrollo emprendedor de un país, sin embargo existen variables externas que inciden de manera positiva o negativa dentro del proceso, por esta razón es importante contextualizar la educación del emprendedor de acuerdo a las diferentes regiones, revisando de manera constante el sistema emprendedor en pro de las necesidades de cada región, puesto que están cambian de acuerdo a la necesidad de los individuos colectivizados, de esta manera se consigue la educación con sostenibilidad.

\section{Áreas en la política de desarrollo empren- dedor a nivel latinoamericano}

Al realizar la visión integral del emprendedor no se puede dejar de lado, las acciones que están desarrollando nuestro entorno, por esta razón en esta parte del trabajo se revisa las acciones que están realizando en el tema de mejoramiento del entorno para emprendedor, de esta manera, se fortalece los planteamientos anteriores, encontrados en el siguiente cuadro: 


\section{Áreas de trabajo para promover} la empresarialidad en América Latina

- Ampliar la base social y de género de la cual surgen los emprendimientos dinámicos.

- Expandir el número y la calidad de las oportunidades de negocios.

- Facilitar el acceso de los potenciales emprendedores a la experiencia de trabajo.

- Favorecer el desarrollo de los equipos emprendedores y sus redes de contactos.

- Mejorar el acceso al financiamiento.

- Fortalecer el proceso emprendedor en las áreas locales.

- Aprovechar el potencial de transformación de los negocios basados en el conocimiento.

- Generar condiciones de entorno más favorables para el crecimiento de las nuevas empresas.

- Adoptar un enfoque sistémico, basado en la complementación de esfuerzos entre distintas áreas y niveles de gobierno, con un fuerte protagonismo del sector privado.

- Asumir el desarrollo emprendedor como una inversión social con visión de largo plazo.

Fuente: Hugo Kantis, América Latina y la experiencia internacional, p. 133.

Con base en este cuadro se presenta un análisis de cada una de las variables en contexto de Hugo Kantis.

1. Ampliar la base social y de género de la cual surgen los emprendimientos dinámicos. Los emprendedores latinoamericanos pertenecen a sectores de clase media y media alta y cuentan con un elevado nivel de educación; por consiguiente, provienen de una base social más estrecha Kantis (2004). Hallando que la edad joven del individuo, así como su pertenencia a una familia emprendedora o su nivel formativo, constituyen variables determinantes en la decisión de crear una empresa (Colombo \& Grilli, 2005; Hayton, 2005).

2. Expandir el número y la calidad de las oportunidades de negocios. La menor presencia relativa de empresas en las economías, la fragmentación de los sistemas productivos, los bajos niveles de ingreso per cápita y una elevada inequidad limitan la cantidad y calidad de las oportunidades de negocios para crear nuevas empresas dinámicas en América Latina, sobre todo las que tienen alto contenido tecnológico Kantis (2004). Las cuales pueden ser tanto institutos de investigación e insti- tuciones públicas como empresas de ámbito internacional o local (Suzuki, Kim \& Bae, 2002; Ucbasaran, Westhead \& Wrigh, 2008).

3. Facilitar el acceso de los potenciales emprendedores a la experiencia de trabajo. La experiencia de trabajo en sectores similares o vinculados a la actividad de la nueva empresa constituye una fuente fundamental para la adquisición de la vocación y de las competencias empresariales, así como para el acceso a información relevante, a la tecnología y a contactos de negocios Kantis (2004). Complementando este análisis se encuentra Varela (2011) la Estructura y dinámica productiva, el perfil sectorial y el regional y el tamaño de las empresas e instituciones en las que trabajan las personas pueden contribuir al desarrollo de competencias emprendedoras en la población trabajadora y en la formación de sus redes de relaciones.

4. Favorecer el desarrollo de los equipos emprendedores y sus redes de contactos. La creación de emprendimientos dinámicos requiere un esfuerzo colectivo. No solo hace falta un equipo de emprendedores con capacidades complementarias y especializadas, sino también una red de contactos que complementen los conocimientos, las habilidades y los recursos de los emprendedores Kantis (2004). Promoviendo activamente el acceso a diversas redes que puedan prestar apoyo a las nuevas empresas, por lo que la existencia de estos recursos en el entorno sería una condición para que prolifere el desarrollo. García. (2010, p. 11).

5. Mejorar el acceso al financiamiento. El análisis puso de manifiesto las consecuencias negativas que enfrentan los emprendedores latinoamericanos por un acceso restringido al financiamiento formal Kantis (2004). La existencia de fuentes diversas de financiación representa un factor de elevada relevancia aportado por el entorno, el cual condiciona en muchos casos la factibilidad de la puesta en marcha de una nueva empresa. Concretamente, el papel desempeñado por bancos, empresas de arrendamiento, empresas de capital riesgo, inversores privados o fondos de desarrollo regional ha sido destacado por autores como Collinson y Gregson (2003) y Neck et al., (2004).

6. Fortalecer el proceso emprendedor en las áreas locales. La investigación comprobó las desventajas 
que existen en las áreas locales latinoamericanas. Los programas de desarrollo emprendedor deben incluir componentes de fomento de la empresarialidad orientados a fortalecer las condiciones para crear y hacer crecer las empresas Kantis (2004). La existencia de agentes tecnológicos de apoyo, como los organismos públicos y privados que dan soporte al emprendimiento, universidades y otros agentes sectoriales, como los centros de investigación, servicios de asesoramiento, etc., condicionan las posibilidades de los emprendedores de identificar y explotar nuevos negocios. García, 2010. P. 11

7. Aprovechar el potencial de transformación de los negocios basados en el conocimiento. La presencia de este tipo de emprendimientos es poco frecuente en la región y las condiciones estructurales que enfrentan quienes desean crear este tipo de empresas son menos ventajosas que en el caso del sector convencional Kantis (2004). En palabras de West, Bamford y Marsden (2008) sugieren que si no existen recursos intangibles, tales como los conocimientos sobre cómo hacer uso de los activos físicos para explotar las oportunidades, dichos activos físicos pierden su capacidad para apoyar la creación de nuevas empresas.

8. Generar condiciones de entorno más favorables para el crecimiento de las nuevas empresas. La investigación reveló que los emprendimientos dinámicos latinoamericanos son los que enfrentan condiciones de entorno más desfavorables Kantis (2004). Estos representan un conjunto de recursos tangibles que posee un territorio, entre los que se incluyen las carreteras, el espacio para oficinas, las zonas habilitadas para la instalación industrial, entre otras (Neck et al., 2004).

9. Adoptar un enfoque sistémico basado en la complementación de esfuerzos. Las debilidades identificadas en el contexto emprendedor latinoamericano justifican la importancia de asumir una estrategia basada en una visión integral y sistémica, más allá de que en cada ámbito en el cual se aplique sea fundamental contar con una adecuada evaluación del funcionamiento de los distintos factores que inciden en el sistema de desarrollo emprendedor Kantis (2004). Al respecto, Venkataraman (2004) señala que los recursos tangibles por sí solos no son suficientes para asegurar el desarrollo empresarial de un territorio. Los recursos tangibles requieren de los intangibles y estos, además, deben estar interrelacionados.

10. Asumir el desarrollo emprendedor como una inversión social con visión de largo plazo. Algunos programas pueden mostrar resultados en tiempos más cortos, como por ejemplo, los que dirigen sus esfuerzos al apoyo de los emprendedores con experiencia previa, o a los que ya concibieron su proyecto de empresa o están en sus comienzos Kantis (2004). Situaciones que dependen de la visión o interés de la administración de turno.

Para resumir, lo importante es la convergencia de todos los factores en La promoción de la cultura emprendedora, se debe transmitir por diversos medios a través de numerosas revistas, programas de televisión y suplementos de diarios que llevan el mensaje emprendedor a millones de hogares. Con respecto a esto, lammarino y McCann (2006) indican que no existe un camino lineal 0 determinista para este desarrollo evolutivo, por lo que cada territorio debe desarrollar su propio patrón.

\section{Conclusiones}

La realidad genera cultura, la cultura genera entorno, el entorno genera formas de pensar que se acciona en la personalidad del individuo, que genera rasgos del emprendedor y se demuestra en su comportamiento, desarrollando competencias que facilitan el camino de emprender, bajo este esquema se hace importante cambiar la educación, renovarla y reinventarla con base a las necesidades de cada territorio y realmente hacia donde se necesita, que en este caso es de acuerdo a su región.

Fortalecer el proceso emprendedor en las áreas locales, donde existen desventajas en áreas locales latinoamericanas. Orientado a programas de desarrollo emprendedor deben incluir componentes de fomento de la empresarialidad orientados a fortalecer las condiciones para crear y hacer crecer las empresas. Hugo Kantis, América Latina y la experiencia internacional. 2004 p. 135.

Generar condiciones de entorno más favorables para el crecimiento de las nuevas empresas. Debido a que los emprendimientos dinámicos latinoamericanos son los que enfrentan condiciones de entorno más desfavorables. 
Hugo Kantis, América Latina y la experiencia internacional. 2004 p. 135.

Adoptar un enfoque sistémico basado en la complementación de esfuerzos. Las debilidades identificadas en el contexto emprendedor latinoamericano justifican la importancia de asumir una estrategia basada en una visión integral y sistémica, más allá de que en cada ámbito en el cual se aplique sea fundamental al contar con una adecuada evaluación del funcionamiento de los distintos factores que inciden en el sistema de desarrollo emprendedor. Hugo Kantis, América Latina y la experiencia internacional. 2004 p. 135.

\section{Referencias bibliográficas}

Alexei, T. y Kolvereid, L. Selfemployment intentions among Russian students. Entrepreneurship and Regional Development, 11(3)(1999):269-271.

Amartya Sen; traducción de Esther Rabasco y Luis Toharia. Desarrollo y Libertad. GeoPlaneta, Editorial, S. A., 1/01/2000, $440 \mathrm{pp}$.

Auerswald, P. E., \& Branscomb, L. M. Valleys of death and Darwinian seas: financing the invention to innovation transition in the United States. Journal of Technology Transfer, 28(2003):227-239.

Audet, J. A Longitudinal Study of the Entrepreneurial Intentions of University Students. Trabajo presentado al Frontiers of Entrepreneurship Research, Babson College, Wellesley, 2002.

Barney, J. B. Firm resources and sustained competitive advantage. Journal of Management, 17(1)(1991): 99-120.

Colombo, M. G., \& Grilli, L. Founders' human capital and the growth of new technology- based firms: A competence-based view. Research Policy, 34(2005):795-816.

Cuesta Santos, Armando. Gestión de Competencias. La Habana: Editorial Academia, 2001, p. 93.

Crant, J. M. The proactive personality scale as a predictor of entrepreneurial intentions. Journal of Small Business Management, 34(3)(1996):42-55.

Dehter, Mario. Responsabilidad Social de las Universiddes Hispanoamericanas para la animación de la Cultura Emprendedora Regional. Universidad Nacional de San Martín. Argentina, (2001).

Douglas, E. J. y Shepherd, D. A. Self-Employment as a Career Choice: Atti tudes, Entrepreneurial Intentions, and Utility Maximization. Entrepreneurship Theory and Practice, 26(3)(2002):81-90.

Duarte Tito, Myriam Ruiz Tibana. Emprendimiento, Una Opción Para el Desarrollo. Scientia Et Technica, diciembre. Pereira Colombia: Universidad Tecnológica, XV(43)(2009):326-331.

Dyer, W.G. Jr. Toward a theory of entrepreneurial careers. Entrepreneurship Theory and Practice, 19(19)(1994):7-21.

Fayole, A. y Bruyat, C. A Conceptual and Methodological Framework to Study, Using the Modeling of Complex System, the Foundation and Development Processes of Innovative Business Activities. RENT XVI, 16th Workshop. Conference Proceedings. Noviembre 21-22, Barcelona, España, 2002.

Foss, K., Foss, N. J., Klein, P. G., \& Klein, S. K. The entrepreneurial organization of heterogeneous capital. Journal of Management Studies, 44(7)(2007):1165-1186.

Gibb, A. "Creating the Entrepreneurial University Worldwide: Do we need a wholly diferrent model of entrepreneurship», Int Ent 2005, Naples, 2005.

Gilder, J. The Spirit of the Enterprise. Penguin Books Ltda. 1984.

Green, R.; David, J.; Dent, M. The Russian entrepreneur: a study of psychological characteristics. International Journal of Entrepreneurial Behaviour and Research. 2 (1)(1996).

Herrera Restrepo, D. América Latina y la fenomenología. México: Universidad Pontifícia de México. Colección Investigación UPM, No. 7, 1998.

Husserl, E. El espíritu común (Gemeingeist). Obra póstuma. 1987.

Husserl, E. Problemas fundamentales de la fenomenología. (C. Moreno y J. San Martín, Trads.). Madrid: Alianza. 1994.

Hoyos V., G., y Vargas G., G. La teoría de la acción comunicativa como nuevo paradigma de investigación 
en ciencias sociales: las ciencias de la discusión. Bogotá: Arfo. 1996.

Kantis Hugo. Desarrollo Emprendedor América Latina y la experiencia internacional, editor, con la colaboración de Pablo Angelelli y Virginia Moori Koenig. 2004.

Kaufmann, P.J.; Welsh, D.H.; Bushmarin, N.V. Locus of control and entrepreneurship in Russian Republic. Entrepreneurship Theory and Practice, 20(1995):43-59.

Korunka, C., Frank H., Lueger, M. and Mugler, J. The entrepreneurial personality in the context of resources, environment, and the start up process-Aconfigura tional approach. Entrepreneurship Theory and Practice, 28(2003):23-42.

Levy- Leboyer, Claude. Gestión de las competencias. Barcelona: Ediciones Gestión 2000, 1997.

Mcclelland, D.C. La Sociedad Ambiciosa., Madrid: Ediciones Guadarrama, 1961.

Mertens, Leonard. La Gestión por competencia laboral en la empresa y la formación profesional. CINTERFOR/ OIT documento en línea http://www.cinterfor.org.ur

Mucchieli, A. Les mentalités. Paris: Presses Universitaires de France. Palich, L. \& Bagby, D. (1995). Using cognitive theory to explain entrepreneurial risk-taking: challenging conventional wisdom. Journal of Business Venturing, 10(1985):425-438.

Neck, H. M., Meyer, G. D., Cohen, B., \& Corbett, A. C. An entrepreneurial system view of new venture creation. Journal of Small Business Management, 42(2)(2004):190-208.

Ong J. W.; Hishamuddin, B. I. Revisiting personality traits in entrepreneurship study from resource based perspective, Business Renaissance Quarterly, 3(1)(2008):97-114.

Orrego, Clara Inés. La fenomenología y el emprendimiento artículo revista ciencias estratégicas, 2009.

Pino Martínez, Agustín del. Empleabilidad y competencias: ¿Nuevas Modas? En Psicología del Trabajo y Gestión de Recursos Humanos. Barcelona: Ediciones Gestión 2000, 1997. p. 45-52.

Porter, M.E. La ventaja competitiva de las naciones. Barcelona, España: Plaza y Janés, 1991.
Robbins, S.P. Comportamiento organizacional. Prentice Hall, 8a. Edic. México, 1999.

Robinson, P., Stimpson, D., Huefner, J. y Hunt, H. An attitude approach to the prediction of entrepreneurship. Entrepreneurship Theory and Pratice, 15(4)(1991):13-32.

Ronstadt, R. «The educated Entrepreneur a New Era of entrepreneurial Education is Beginning», American Journal of Small Business, verano, 1985.

Senge, Peter: El arte y la práctica de la organización abierta al aprendizaje - 2ª Ed. Buenos Aires: Granica, 2005.

Sánchez-Almagro, M. L. Perfil Psicológico del Autoempleado. Tesis doctoral no publicada. Madrid, España: Universidad Complutense de Madrid, 2003.

Sánchez, Morrero, Martínez. Una mirada a los orígenes de las competencias laborales, 2005.

Timmons, J. A; Spinelli, S. New Venture Creation Entrepreneurship for The 21 st Centhury, $6^{a}$ edición. Irwin, Boston: McGraw Hill, 2004.

Varela, R. Bedoya O. L. Desarrollo de Empresarios Basado en Competencias Empresariales: El Caso Jóvenes con Empresa, Balas Conference 20066, Lima Perú. Mayo 2006.

Vargas Guillén, G. «Formación y subjetividad». En: Colección «Filosofía y enseñanza de la Filosofía». Bogotá: Universidad Pedagógica Nacional, 2007.

Veciana, J. M. Características del empresario en España. Papeles de Economía Española, núm. 39/40, 1989.

Wernerfelt, B. A resource-based view of the firm. Strategic Management Journal, 15(1984):171-180.

West III, G. P., \& Bamford, C. E. Creating a technologybased entrepreneurial economy: a resource based theory perspective. Journal of Technology Transfer, 30(2005): 433-451.

West III, G. P., Bamford, C. E., \& Marsden, J. W. Contrasting entrepreneurial economic development in emerging Latin American economies: applications and extensions of resource- based Theory. Entrepreneurship Theory and Practice, 32(1)(2008):15-36. 